\title{
Detailed Multi-Dimensional Assessment of Fatigue in Inflammatory Bowel Disease
}

\author{
Kristina Aluzaite $^{a}$ Rashid Al-Mandhari ${ }^{a}$ Hamish Osborne ${ }^{a}$ Christine Ho $^{a}$ b \\ Merrilee Williams $^{a}$ b Marie-Michelle Sullivan ${ }^{a} \quad$ Catherine E. Hobbs $^{a}$ \\ Michael Schultz ${ }^{a, b}$ \\ ${ }^{a}$ Department of Medicine, University of Otago, Dunedin, New Zealand; ${ }^{b}$ Gastroenterology Unit, Southern District \\ Health Board, Dunedin Public Hospital, Dunedin, New Zealand
}

\section{Keywords}

Crohn's disease · Fatigue - Inflammatory bowel diseases .

Quality of life $\cdot$ Ulcerative colitis

\begin{abstract}
Background: Fatigue is a symptom commonly reported by patients with inflammatory bowel disease (IBD). Treating any underlying inflammation in active disease improves the health outcomes and decreases fatigue, but fatigue still persists in remission, negatively affecting patients' quality of life and posing a challenge for the treating physician. The aim of this study was to describe the prevalence of fatigue in patients with IBD and investigate possible contributing factors. Methods: Recruited IBD patients from the Otago region in southern New Zealand were asked to complete demographic, physical activity (IPAQ) and fatigue questionnaires (Brief Fatigue Inventory, Multidimensional Fatigue Inventory). Disease activity and factors contributing to fatigue were assessed through self-reporting and laboratory biomarkers. Results: One hundred and thirteen of the contacted 469 IBD patients participated in the study. Depending on the questionnaire used, the prevalence of fatigue in IBD was high in remission $(39.5-44.2 \%)$ but significantly higher $(p<0.001)$ in
\end{abstract}

() 2019 S. Karger AG, Basel active disease (80.0-82.9\%). Several factors such as age, disease duration, level of physical activity, gender and diet were found to be associated with increased fatigue and were attributed to either mental or physical fatigue categories. Multifactorial Fatigue Inventory provided insights into different types of fatigue, and revealed a significant mental fatigue component in both active and remission disease patients. Iron deficiency was not associated with fatigue levels. Conclusions: Fatigue in IBD is multi-faceted and highly prevalent in both active and remission IBD. Further investigations, addressing the complexity of the symptom and its reporting are needed.

๑ 2019 S. Karger AG, Basel

\section{Background}

Fatigue is a common and persistent symptom of a number of chronic conditions, such as cancers, rheumatoid arthritis, and has been reported to be problematic in inflammatory bowel diseases (IBD) [1-3]. The most com-

\section{K. Aluzaite and R. Al-Mandhari contributed equally to this work.}

\section{KARGER}

E-Mail karger@karger.com

www.karger.com/iid
Prof. Michael Schultz, MD, PhD, FRACP

Department of Medicine, Dunedin School of Medicine

University of Otago, PO Box 913

Dunedin 9054 (New Zealand)

E-Mail michael.schultz@ otago.ac.nz 
mon forms of IBD are Crohn's disease (CD) and ulcerative colitis (UC) [4]. Both forms are characterised by intermittent "flare-ups" with increased inflammatory activity, along with episodes of remission, with reduced inflammatory activity $[5,6]$.

Fatigue has been described as an unpleasant, distressing [7] and persistent tiredness, weakness or exhaustion at physical, mental or both levels. It is also known as subjective, variable and multifactorial [8]. Fatigue is one of the most frequent symptoms in IBD and has been reported to be present in $44-86 \%$ of patients with active disease, and up to $22-48 \%$ of those in remission $[2,8]-$ substantially higher than in the healthy population $[7,9]$. Due to the underlying complexity of factors contributing to fatigue, treatments are often empirical and resolution of symptoms difficult to achieve.

Fatigue in IBD has been attributed to such factors as disease activity $[10,11]$ and severity of IBD symptoms $[12,13]$, duration of illness [12], gender [9, 14], psychological [15] and lifestyle factors, while other studies found fatigue to be independent of these variables $[10,16]$. Depleted iron stores are frequently associated with fatigue in a number of conditions, and are a common side effect of IBD [17]. However, determining iron deficiency in IBD is complex, as the serum markers are affected by the inflammatory mediators [18].

IBD in remission is associated with decreased levels of fatigue potentially due to reduced inflammation, but the prevalence remains substantial, the specific causes unknown and management difficult [8].

Furthermore, there is a lack of consensus on what defines fatigue and how to measure it, and several tools have been used to capture the complexity $[7,19]$. Most commonly used are the Brief Fatigue Inventory (BFI) [20], Multifactorial Fatigue Inventory (MFI) [21], Functional assessment of chronic illness therapy-fatigue (FACIT-F) [22], Fatigue questionnaire (FQ) [23] along with many more. However, none of these questionnaires was developed in IBD populations. We chose a one-dimensional BFI questionnaire that offers a quick fatigue assessment, and the MFI - a more in-depth, multifactorial description of fatigue $[19,21]$. These tools were chosen due to their frequent use to assess fatigue in IBD disease studies [2, 7 , $10,13,14,24-26]$ and because they represented two different approaches to fatigue measurement. To date, there have been very few studies that focused on fatigue in IBD as the primary outcome measure [2], and explored the symptom in its multi-dimensional nature. There is also lack of data on the influence of lifestyle variables on the levels of fatigue in IBD patients.
The aim of this study was therefore primarily to determine the prevalence of fatigue in IBD patients in Dunedin, New Zealand, and secondarily to investigate in detail associated demographic, clinical and lifestyle variables in both active disease and disease in remission. Finally, we wanted to explore the usefulness of the questionnaires in clinical practice.

\section{Methods}

\section{Patient Recruitment}

Adult patients ( $\geq 18$ years old), with histologically confirmed IBD were identified through the Dunedin Public Hospital IBD outpatient clinics and the hospital's Gastroenterology Unit, IBDspecific database (EpiSoft, Sydney, Australia). Following consent, patients were asked to complete several questionnaires to describe their demographic variables, IBD disease activity, perceived fatigue, level of physical activity, and to provide blood and stool samples. Patients completed the questionnaires independently.

\section{Assessment of Inflammatory Activity}

Patients were asked to complete a disease activity questionnaire specific to their disease diagnosis (Crohn's Disease Activity Index [CDAI] [27] and Simple Clinical Colitis Activity Index [SCCAI] [28] for $\mathrm{CD}$ and $\mathrm{UC}$ patients, respectively). C-reactive protein (CRP), and faecal calprotectin (FCP) [29] were measured in all patients (at the Southern Community Laboratories, Dunedin, New Zealand).

Definition of remission in IBD is controversial and the commonly used disease activity markers have limitations in categorising patients to active and disease in remission, as specific cut-off values are difficult to determine. Patients were defined to have active disease if at least one of the following measures was above the pre-defined values: CDAI $>150$ [30] or SCCAI $>5$ [31], FCP $>150$ $\mu \mathrm{g} / \mathrm{g}[32], \mathrm{CRP}>5 \mathrm{mg} / \mathrm{L}[33,34]$, as suggested by a number of studies. Patients at or below these values were considered to be in remission [35-37]. In order to represent an accurate picture of remission in IBD, we also separately reported fatigue prevalence in patients with a CRP $<10 \mathrm{mg} / \mathrm{L}$, as suggested by the latest ECCO guidelines [29].

\section{Fatigue}

To measure fatigue in patients, the BFI and Multidimensional Fatigue Inventory (MFI) were used. The definition of fatigue was complicated by lack of clear cut-off scores; hence, values reported in other studies were used $[2,20]$. The BFI is a single dimension fatigue questionnaire, with values $>4$ indicating moderate fatigue and $>6$ severe fatigue [20]. The MFI consists of multiple dimensions - General Fatigue, Physical Fatigue, Reduced Activity, Reduced Motivation, Mental Fatigue; each dimension contains 4 questions and results in scores of 4-20, increasing with fatigue. We considered values $>10$ moderate and $>14$ as severe fatigue in each of the dimensions; cumulative scores $>50$ points for overall MFI indicated moderate to severe and $>70$ severe fatigue $[2,14]$. All scores were reported to describe the prevalence of fatigue. 
Table 1. Study population characteristics - demographic, clinical and lifestyle variables

\begin{tabular}{|c|c|c|c|c|}
\hline Characteristic & $\begin{array}{l}\text { Total sample } \\
(n=113)\end{array}$ & $\begin{array}{l}\text { Active } \\
(n=70)\end{array}$ & $\begin{array}{l}\text { Remission } \\
(n=43)\end{array}$ & $\begin{array}{l}p \\
\text { (active vs. remission) }\end{array}$ \\
\hline Age, years & $47.2(16.6)$ & $44.8(15.7)$ & $51.1(17.5)$ & $0.57^{\mathrm{a}}$ \\
\hline Type of IBD & & & & $0.040^{\mathrm{b}, *}$ \\
\hline CD & $70(61.9 \%)$ & $49(70 \%)$ & $21(48.8 \%)$ & \\
\hline UC & $43(38.1 \%)$ & $21(30 \%)$ & $22(51.2 \%)$ & \\
\hline $\mathrm{BMI}$ & $25.6(22.7-29.3)$ & $25.0(22.6-29.4)$ & $26.5(23.2-29.1)$ & $0.58^{\mathrm{c}}$ \\
\hline Following a diet, yes & $23(20.4 \%)$ & 15 (21.4\%) & $8(18.6 \%)$ & $0.90^{\mathrm{b}}$ \\
\hline Supplements, yes & $42(37.2 \%)$ & $27(38.6 \%)$ & $15(34.9 \%)$ & $0.85^{\mathrm{b}}$ \\
\hline \multicolumn{5}{|l|}{ Physical activity } \\
\hline Low & $27(23.9 \%)$ & $18(25.7 \%)$ & $9(20.9 \%)$ & $0.60^{\mathrm{b}}$ \\
\hline Moderate & $51(45.1 \%)$ & $29(40.0 \%)$ & $22(51.2 \%)$ & \\
\hline Iron deficiency, yes & $54(47.8 \%)$ & $47(67.1 \%)$ & $7(16.3 \%)$ & \\
\hline SCCAI score & & $6.5(5.75-7.25)$ & $3.0(2.0-5.0)$ & \\
\hline CDAI score & & $125.0(83.3-164.5)$ & $67.0(50.0-91.0)$ & \\
\hline $\mathrm{CRP}, \mathrm{mg} / \mathrm{L}$ & & $4.0(1.25-7.0)$ & $1.0(1.0-2.5)$ & \\
\hline $\mathrm{FCP}, \mu \mathrm{g} / \mathrm{L}$ & & $158.5(58.5-406.0)$ & $5.0(0.0-61.0)$ & \\
\hline
\end{tabular}

Data are presented as mean (SD), $n$ (\%) or median (IQR). ${ }^{\mathrm{a}} t$ test; ${ }^{\mathrm{b}}$ Pearson's $\chi^{2}$ test; ${ }^{\mathrm{c}}$ Mann-Whitney-Wilcoxon; * statistically significant.

\section{Physical Activity}

The International Physical Activity Questionnaire (IPAQ) [38] was used to estimate participants' level of physical activity. Values lower than 600 were considered low physical activity, 6003,000 - moderate, while $>3,000$ - high physical activity. Participants also reported the number of hours they spend per day sitting, which was also used as an indication of physical activity and a lifestyle measure.

\section{Iron Deficiency}

Serum ferritin was used to assess blood iron status (all at the Southern Community Laboratories, Dunedin, New Zealand). Iron deficiency diagnostic criteria depend on inflammation levels [17]; and according to the ECCO guidelines for Iron Deficiency and Anaemia in IBD [17], adult serum ferritin values less than $30 \mu \mathrm{g} / \mathrm{L}$ were considered indicative of iron deficiency in IBD patients in remission and serum ferritin values of even $<100 \mu \mathrm{g} / \mathrm{L}$ could be indicative of possible iron deficiency in active disease [39]. Patients were assigned presence of iron deficiency accordingly.

\section{Statistical Considerations}

Medians and interquartile ranges (IQR) and means and standard deviations (SD) were calculated to describe the study sample. Sample characteristics were compared between patients with active and quiescent disease using Pearson's $\chi^{2}$ test for categorical variables, Mann-Whitney-Wilcoxon and $t$ tests test for continuous data. Simple linear regression method was used to select explanatory variables for general multiple linear regression to model con- tinuous fatigue outcome measures. Type of IBD was included in all the models due to the expected clinical impact. Backwards selection was used to select informative explanatory variables. Pairwise interactions between disease activity status, type of IBD, gender and presence of iron deficiency were tested. Diagnostic plots and model fit values were used to select the best fit models.

Spearman correlations were used to explore relationship between disease activity markers and fatigue questionnaire scores. $p$ values lower than 0.05 were considered statistically significant. Analyses were performed using $\mathrm{R}$ statistical computing language [40].

\section{Results}

\section{Study Sample (Table 1)}

Of the contacted IBD patients, $24.1 \%(n=113 / 469)$ participated in the study (Fig. 1). 61.6\% $(n=69)$ of them were female with mean (SD) 47.2 (16.6) years of age. $61.9 \%(n=70)$ and $38.1 \%(n=43)$ of the participants had $\mathrm{CD}$ and UC, respectively. According to the criteria defined above and with a CRP cut-off at $<5 \mathrm{mg} / \mathrm{L}, 70 \%(n=$ 49) CD patients had active disease and $30 \%(n=21)$ were in remission at the time of the study, while $49 \%(n=21)$ and $51 \%(n=22)$ UC patients had active disease or were 
Fig. 1. Study participant recruitment and

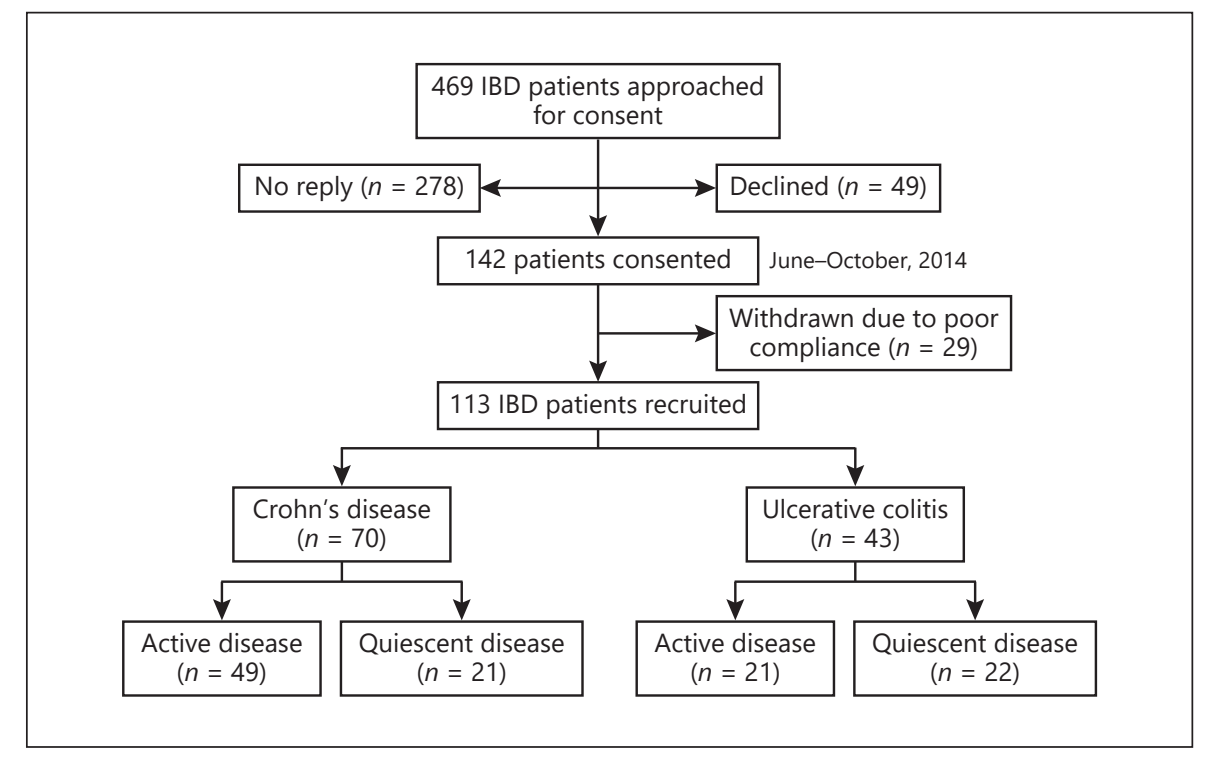
response.

in remission, respectively (Fig. 1). Significantly more CD patients had active disease than UC $(p=0.04)$. Median (IQR) duration of disease was significantly longer in the active disease group (13.5 years [6.3-22.8]) than in patients in remission (8.0 years $[4.0-16.5])(p=0.026)$. $47.8 \%(n=54)$ of the study participants had iron deficiency as indicated by their serum ferritin levels.

Median (IQR) BMI was 25.6 (22.7-29.3); 20.4\% ( $n=$ $23)$ and $37.2 \%(n=42)$ followed some diet and used supplements, respectively. Finally, majority $(76.1 \%)$ of the participants were moderately or highly physically active.

\section{Levels of Fatigue in IBD Patients}

The proportion of IBD patients with active disease that experienced moderate or severe fatigue according to BFI and MFI overall questionnaires was 82.9 and $80.0 \%$, respectively. In contrast but still clinically important, 39.5 and $44.2 \%$ of the quiescent disease IBD patients experienced moderate or severe fatigue according to $B F I$ and MFI overall questionnaires, respectively.

Based on BFI and all MFI dimension results, 59.2$83.7 \%$ of active CD and $14.3-71.4 \%$ of remission CD patients reported moderate to severe fatigue; $42.9-95.2 \%$ of active UC and $22.7-54.5 \%$ of remission UC patients reported moderate to severe fatigue (Fig. 2 and online suppl. Table 1; see www.karger.com/doi/10.1159/000496054 for all online suppl. material).

There was a large variation between the different fatigue dimensions in all patient categories, highlighting the multifactorial nature of this symptom. The most prevalent type of moderate and severe fatigue in all studied patients was General Fatigue (71.4-95.2\%). The least prevalent was Mental Fatigue in CD and UC active disease (42.9-59.2\%), and Reduced Motivation (14.3\%) and Reduced Activity (22.7\%) in CD and UC in remission, respectively (Fig. 2).

There was a larger difference between severe fatigue prevalence in disease in remission comparing to active disease (median 2.95 times lower) than in moderate fatigue (median 1.1 times lower) (Fig. 2). There was little difference between Reduced Activity, Mental Fatigue and Physical Fatigue prevalence between active disease patients and patients in remission.

It is noteworthy that all fatigue measures (except for MFI Mental Fatigue: $p=0.064$ ) indicated statistically significantly higher fatigue levels in active IBD compared to remission $(p<0.001)$ (Table 2$)$.

\section{Using Different Disease Indices (Table 3)}

Increasing the threshold for quiescent disease to CRP $<10 \mathrm{mg} / \mathrm{L}$ according to ECCO guidelines resulted in 61 active (41 CD and $20 \mathrm{UC}$ ) and 52 (29 CD and $23 \mathrm{UC}$ ) patients in remission (Table 3 ). Using these categories of active and IBD in remission, the prevalence of fatigue was not significantly different from the more conservative (CRP $<5 \mathrm{mg} / \mathrm{L}$ ) disease in remission definition.

\section{Variables Associated with Fatigue}

Multiple linear regression slope coefficients and 95\% confidence intervals along with explanatory variable and model $p$ values are listed in Table 4 . 


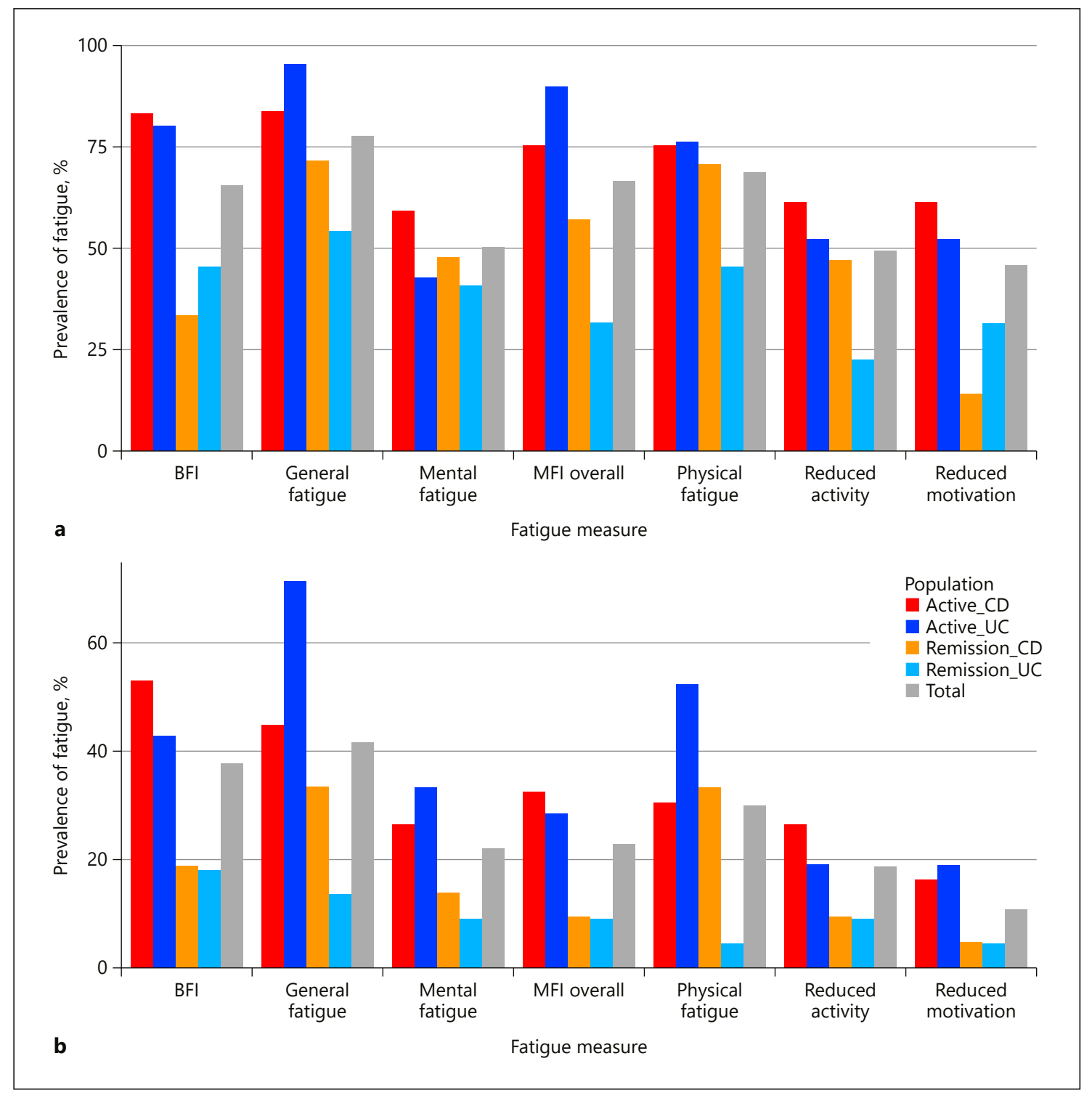

Fig. 2. Comparison of population percentages experiencing moderate and severe fatigue (a) and severe fatigue (b).

IBD disease status was a highly significant explanatory variable; patients with active disease reported higher fatigue scores in BFI and across the MFI dimensions $(p<$ 0.001 ), except for the Mental Fatigue score. Fatigue scores (MFI overall) were significantly positively correlated with self-reported CDAI and SCCAI scores (Spearman's rho = $0.41, p<0.001$, and rho $=0.54, p<0.001$, respectively), in comparison to laboratory inflammation measures FCP and CRP (Fig. 3).

Females scored significantly higher in MFI General Fatigue ( $p=0.008)$, Physical Fatigue $(p<0.001)$, Reduced Activity $(p=0.023)$ and overall MFI measures $(p=0.011)$.
There was some evidence that female gender was also linked with higher BFI scores $(p=0.052)$.

Dietary supplement users had lower Mental Fatigue $(p=0.042)$ and overall MFI scores $(p=0.019)$. Shorter duration of the disease was significantly associated with greater fatigue according to BFI $(p=0.002)$ and MFI General Fatigue dimension $(p=0.009)$. Similarly, older age patients experienced lower Mental Fatigue $(p=0.006)$.

Higher level of physical activity was associated with lower Physical $(p=0.040)$ and Mental $(p=0.006)$ fatigue levels, as well as lower MFI overall, Reduced Activity, Reduced Motivation scores $(p=0.004-0.040)$. There was 
Table 2. Study sample fatigue measures: remission versus active disease

\begin{tabular}{lcccc}
\hline Fatigue measure score & $\begin{array}{l}\text { Total sample } \\
(n=113)\end{array}$ & $\begin{array}{l}\text { Active } \\
(n=70)\end{array}$ & $\begin{array}{l}\text { Remission } \\
(n=43)\end{array}$ & $\begin{array}{l}p \text { value } \\
(\text { active vs. remission) }\end{array}$ \\
\hline BFI & $4.9(2.3)$ & $5.7(1.8)$ & $3.6(2.3)$ & $<0.001^{*}$ \\
MFI overall & $56.9(17.3)$ & $62.3(15.6)$ & $48.0(16.2)$ & $<0.001^{*}$ \\
MFI General Fatigue & $13.6(3.9)$ & $14.8(3.4)$ & $11.6(3.8)$ & $<0.001^{*}$ \\
MFI Physical Fatigue & $12.3(3.8)$ & $13.2(3.7)$ & $10.8(3.6)$ & $0.0011^{*}$ \\
MFI Reduced Activity & $11.0(4.0)$ & $12.0(3.9)$ & $9.4(3.7)$ & $<0.001^{*}$ \\
MFI Reduced Motivation & $10.1(3.6)$ & $11.1(3.5)$ & $8.6(3.1)$ & $<0.001^{*}$ \\
MFI Mental Fatigue & $10.7(4.3)$ & $11.3(4.4)$ & $9.8(3.9)$ & 0.064 \\
\hline
\end{tabular}

Data are presented as mean (SD). * Statistically significant.

Table 3. Comparison of fatigue prevalence using different values of CRP as one of categorizing disease indices

\begin{tabular}{|c|c|c|c|c|}
\hline Moderate/severe & $52(85.2 \%)$ & $22(42.3 \%)$ & $57(81.4 \%)$ & $17(39.5 \%)$ \\
\hline Severe & $32(52.5 \%)$ & $11(21.2 \%)$ & $35(50.0 \%)$ & $8(18.6 \%)$ \\
\hline Severe & $21(34.4 \%)$ & $5(9.6 \%)$ & $22(31.4 \%)$ & $5(11.6 \%)$ \\
\hline
\end{tabular}

Data are presented as median (IQR) or $n(\%)$.

some evidence that more hours spent sitting in a day was linked with higher MFI Reduced Activity and Reduced Motivation scores ( $p=0.065-0.085)$. There was no association between presence of iron deficiency and different fatigue measures.

There was a significant interaction between disease activity status and type of IBD, with CD patients experiencing a smaller increase (2-point increase vs. 5.2-point increase in UC patients) in the MFI General Fatigue measure $(p=0.023)$. There were no other significant interactions between disease activity status, gender, type of IBD and presence of iron deficiency.

\section{Discussion}

$\mathrm{CD}$ and UC are multi-factorial, systemic diseases that are characterised by episodes of active and quiescent disease. Patients with IBD commonly experience fatigue, and while it is often attributed to active inflammation, even patients in remission report symptoms of fatigue. The aim of this study was to estimate and characterise fatigue in an IBD population and to identify potential contributing factors.

We used two fatigue inventories to assess fatigue in our population, and identified considerable variation between their estimates. While the BFI produced a combined score, the MFI allowed us to separate and measure different subtypes of fatigue in the study population. Notably, while the prevalence of severe fatigue was lower in quiescent disease than in active disease, moderate fatigue scores persisted in the study population in remission.

We identified a high prevalence of moderate to severe fatigue in both active and quiescent disease patients (82.9-80.0\% of active and $39.5-44.2 \%$ of remission, based on BFI and MFI overall scores, respectively) comparable with previous findings $[2,8]$. While fatigue was significantly higher in active than in quiescent disease, the latter was still substantially higher than that in a healthy general population $[7,9]$, highlighting an important clinical 


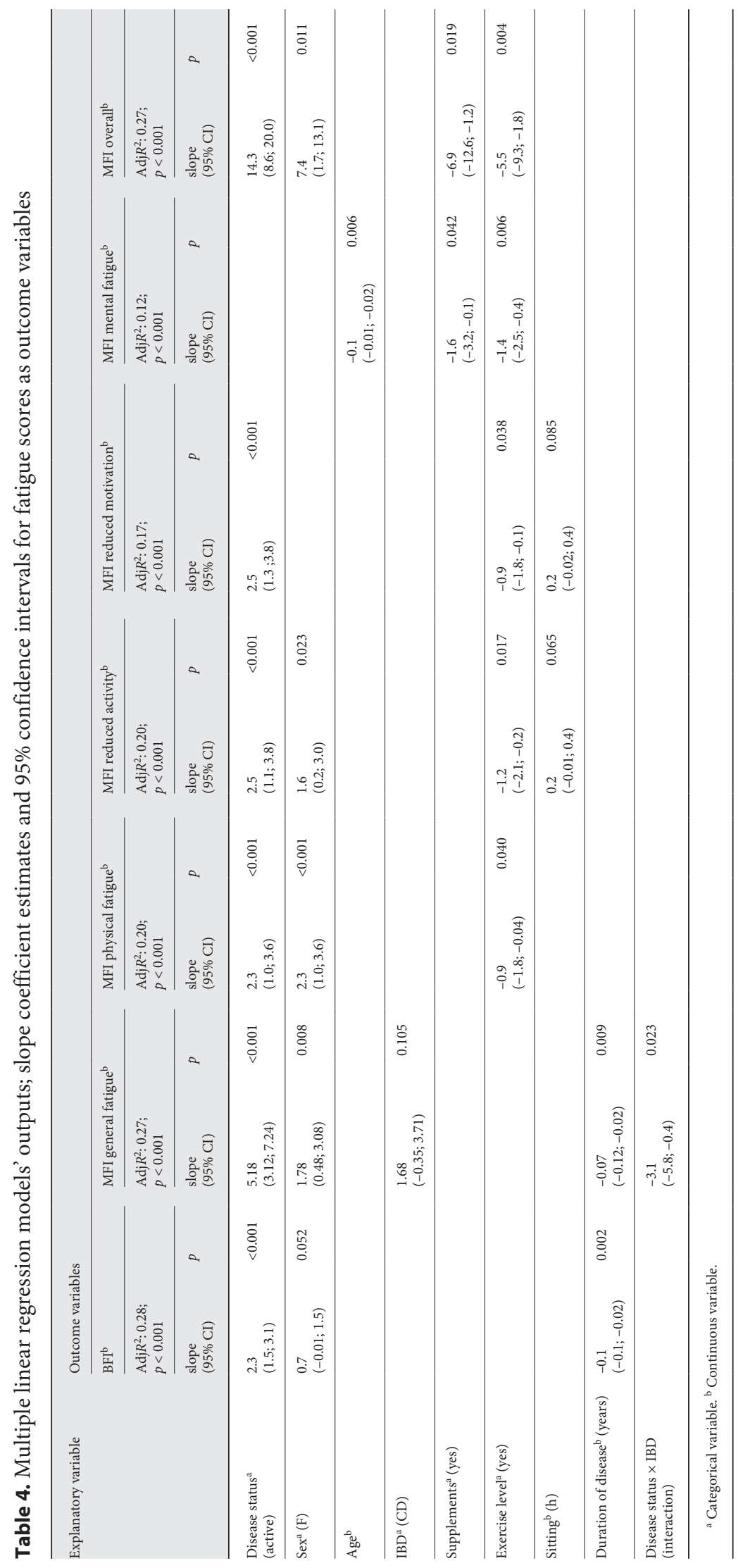




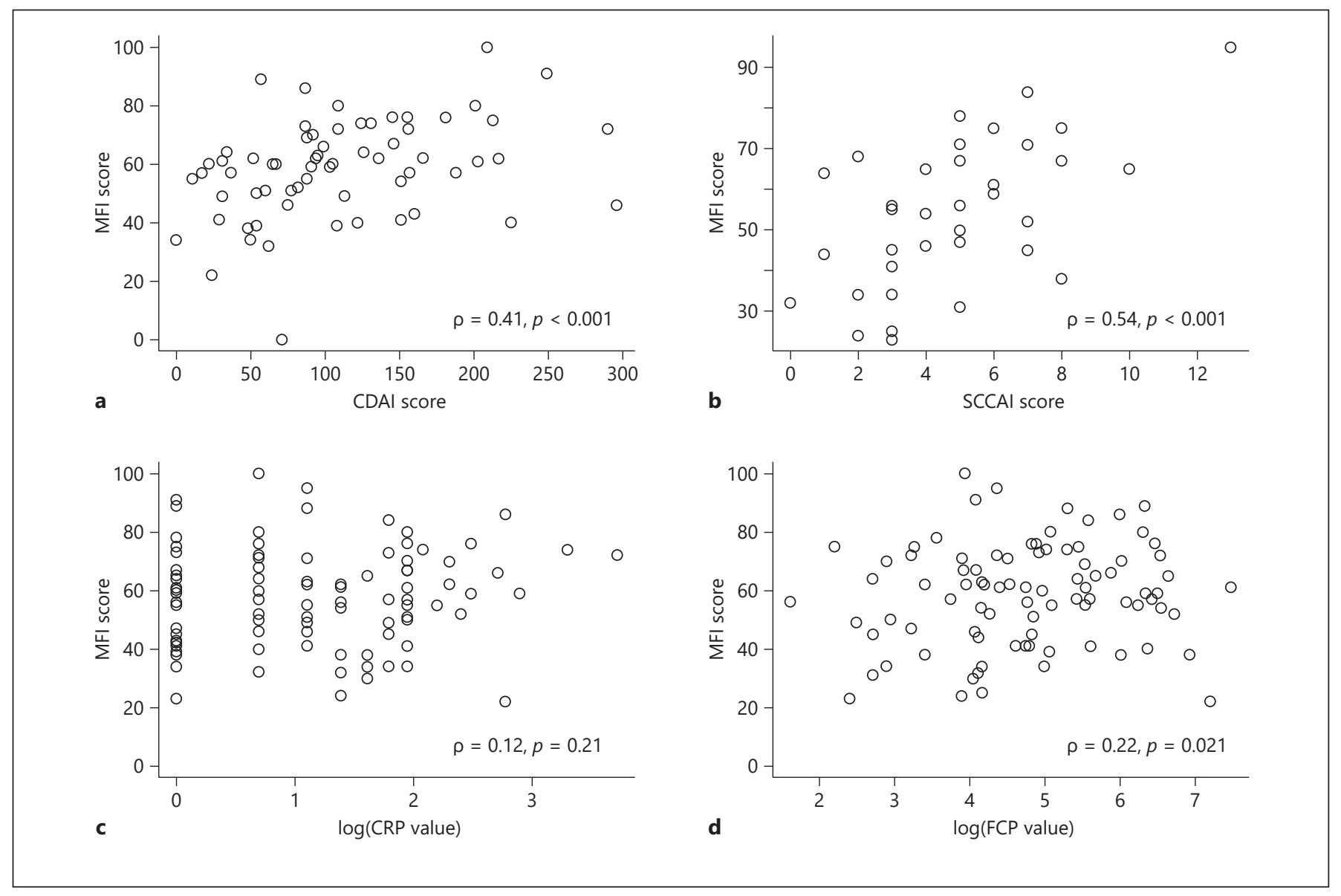

Fig. 3. Correlation of MFI overall with disease activity diagnostic indices Crohn's disease (CD) CDAI score (a), ulcerative colitis (UC) SCCAI score (b), combined CD and UC CRP scores $\left(\log _{10}\right)(\mathbf{c})$, and combined CD and UC FCP values $\left(\log _{10}\right)(\mathbf{d})$.

challenge. Disease activity status was strongly linked with higher fatigue levels across most fatigue dimensions.

We explored a large number of possible explanatory variables associated with fatigue. Notably, some factors could be attributed to physical and mental fatigue categories. Level of physical activity and number of hours spent sitting significantly influenced the mental aspects of fatigue, suggesting that exercise could be a valuable tool to alleviate this. We also found a strong negative association between fatigue and the duration of the disease; patients who had the disease for longer period of time reported lower general fatigue. The same was observed for older participants - they had significantly lower mental fatigue. This could be explained by the adjustment to disease symptoms and the lifestyle impact on perceived fatigue. Similarly, in other studies increased fatigue has been found to be consistently associated with stress [15], de- pression, anxiety [25], while some studies [2] indicate improvements in fatigue levels after mental therapy [41, 42] that support the focus required on the mental aspect of fatigue. Finally, female gender was associated with higher fatigue levels across the fatigue dimensions.

Anaemia and iron deficiency are common side effects of IBD, mainly in active disease, and a number of studies have shown the association with fatigue in IBD patients. Our dataset did not capture association between presence of iron deficiency and fatigue levels; larger sample size would potentially be required for further investigations.

A detailed analysis of fatigue in active and quiescent disease is complicated due to the continuous nature of IBD symptoms and disease activity, and resulting loosely defined disease state-differentiating scores. In order to eliminate inflammation as a confounding factor to assess fatigue in patients in remission, we chose a more conser- 
vative definition of remission (CDAI $\leq 150$, SCCAI $\leq 5$, FCP $\leq 150 \mu \mathrm{g} / \mathrm{g}$ and CRP $\leq 5 \mathrm{mg} / \mathrm{L}$ ), but also made comparisons using a higher CRP cut-off score (CRP $<10$ $\mathrm{mg} / \mathrm{L}$ ) as suggested by the ECCO guidelines [29]. A recent study by Vogelaar et al. [43] identified statistically significant distinct immune profiles between fatigued and non-fatigued IBD patients in remission, as indicated by their FCP and CRP values, which in combination with our findings may suggest an important role of subclinical inflammation in fatigue prevalence.

Our findings support the use of a multidimensional fatigue inventory, such as MFI, as it allows capturing higher resolution image of fatigue that may enable us to narrow the problem and initiate a more directed treatment. However, the BFI is faster, and has been reported to be a good choice in patients with severe disease. The overall scores identifying presence of fatigue in both questionnaires were similar in our study population. Furthermore, fatigue cut-off scores require further validation and do not necessarily represent the qualitative descriptions of fatigue, especially since neither BFI nor MFI has been validated in IBD patients.

The main limitation of our study was the relatively small sample size. Due to complex and multi-faceted nature of the study topic, in addition to likely variation and bias of the reported fatigue values, larger sample sizes are required to establish stronger associations between variables. Moreover, participants completed the questionnaires and disease activity scores on their own, which means there might have been a possibility for questionnaire completion error.

Our study population might also not be reflective on the entire IBD patient population, since study participants were recruited from a specialist treatment unit, and only $30 \%$ of approached patients agreed to participate. It is also likely that patients experiencing higher levels of fatigue did not agree to participate; therefore, our results may underestimate true fatigue rates. The opposite is also possible, as patients with no or low levels of fatigue may have not been interested to participate.

\section{Conclusions}

In summary, we confirmed the expected high fatigue prevalence in our IBD population, and identified alarming levels of fatigue in patients in remission. Our study findings highlight the complexity and variability of fatigue presentation in IBD population, and address the need for further studies and consensus of fatigue measurements. Active disease status and female gender were highly statistically significantly linked with increased fatigue. There was evidence that higher physical activity may result in lower mental aspects of fatigue, as well as longer disease duration and increasing age may reduce the experience of fatigue. Surprisingly, we did not identify significant association between serum ferritin values and increased levels of fatigue. Further investigations with larger groups of patients are required to explore the full scope of the problem and validate associated explanatory variables.

\section{Acknowledgements}

This work was supported by GutHealthNetwork, a research theme of the University of Otago. Rashid Al-Mandhari received a scholarship from the University of Otago.

\section{Statement of Ethics}

This study was approved by the New Zealand Health and Disability Ethics Committee (reference number 14/CEN/67) that complies with the Declaration of Helsinki standards. All study participants have given a written informed consent.

\section{Disclosure Statement}

The authors declare no conflicts of interest.

\section{References}

1 Casati J, Toner BB, de Rooy EC, Drossman DA, Maunder RG. Concerns of patients with inflammatory bowel disease: a review of emerging themes. Dig Dis Sci. 2000 Jan;45(1): 26-31.

2 Czuber-Dochan W, Ream E, Norton C. Review article: description and management of fatigue in inflammatory bowel disease. Aliment Pharmacol Ther. 2013 Mar;37(5):50516.
3 Singh S, Blanchard A, Walker JR, Graff LA, Miller N, Bernstein CN. Common symptoms and stressors among individuals with inflammatory bowel diseases. Clin Gastroenterol Hepatol. 2011;9(9):769-75.

4 Baumgart DC. Crohn's Disease and Ulcerative Colitis-From Epidemiology and Immunobiology to a Rational Diagnostic and Therapeutic Approach. New York: Springer; 2012. 
5 Gaya DR, Lyon TD, Duncan A, Neilly JB, Han $S$, Howell J, et al. Faecal calprotectin in the assessment of Crohn's disease activity. QJM. 2005;98(6):435-41.

6 Hanauer SB. Inflammatory bowel disease: epidemiology, pathogenesis, and therapeutic opportunities. Inflamm Bowel Dis. 2006 Jan; 12 Suppl 1:S3-9.

7 Minderhoud IM, Oldenburg B, van Dam PS, van Berge Henegouwen GP. High prevalence of fatigue in quiescent inflammatory bowel disease is not related to adrenocortical insufficiency. Am J Gastroenterol. 2003 May;98(5): 1088-93.

8 van Langenberg DR, Gibson PR. Systematic review: fatigue in inflammatory bowel disease. Aliment Pharmacol Ther. 2010 Jul; 32(2):131-43

9 Simrén M, Svedlund J, Posserud I, Bjornsson ES, Abrahamsson H. Predictors of subjective fatigue in chronic gastrointestinal disease. $\mathrm{Al}-$ iment Pharmacol Ther. 2008 Sep;28(5):63847.

10 Bager P, Befrits R, Wikman O, Lindgren S, Moum B, Hjortswang $\mathrm{H}$, et al. Fatigue in outpatients with inflammatory bowel disease is common and multifactorial. Aliment Pharmacol Ther. 2012 Jan;35(1):133-41.

11 Yoo S, Jung YS, Park JH, Kim HJ, Cho YK, Sohn CI, et al. Fatigue severity and factors associated with high fatigue levels in Korean patients with inflammatory bowel disease. Gut Liver. 2014 Mar;8(2):148-53.

12 Jelsness-Jørgensen LP, Bernklev T, Henriksen $\mathrm{M}$, Torp R, Moum BA. Chronic fatigue is more prevalent in patients with inflammatory bowel disease than in healthy controls. Inflamm Bowel Dis. 2011 Jul;17(7):1564-72.

13 Minderhoud IM, Samsom M, Oldenburg B. Crohn's disease, fatigue, and infliximab: is there a role for cytokines in the pathogenesis of fatigue? World J Gastroenterol. 2007 Apr; 13(14):2089-93.

14 Romberg-Camps MJ, Bol Y, Dagnelie PC, Hesselink-van de Kruijs MA, Kester AD, Engels LG, et al. Fatigue and health-related quality of life in inflammatory bowel disease: results from a population-based study in the Netherlands: the IBD-South Limburg cohort. Inflamm Bowel Dis. 2010 Dec;16(12):213747.

15 Tanaka M, Kazuma K. Ulcerative colitis: factors affecting difficulties of life and psychological well being of patients in remission. J Clin Nurs. 2005 Jan;14(1):65-73.

16 Römkens TE, van Vugt-van Pinxteren MW, Nagengast FM, van Oijen MG, de Jong DJ. High prevalence of fatigue in inflammatory bowel disease: A case control study. J Crohn's Colitis. 2011 Aug;5(4):332-7.

17 Dignass AU, Gasche C, Bettenworth D, Birgegård G, Danese S, Gisbert JP, et al.; European Crohn's and Colitis Organisation [ECCO]. European consensus on the diagnosis and management of iron deficiency and anaemia in inflammatory bowel diseases. J Crohn’s Colitis. 2015 Mar;9(3):211-22.
18 Packer N, Hoffman-Goetz L, Ward G. Does physical activity affect quality of life, disease symptoms and immune measures in patients with inflammatory bowel disease? A systematic review. J Sports Med Phys Fitness. 2010 Mar;50(1):1-18.

19 Dittner AJ, Wessely SC, Brown RG. The assessment of fatigue: a practical guide for clinicians and researchers. J Psychosom Res. 2004 Feb;56(2):157-70.

20 Mendoza TR, Wang XS, Cleeland CS, Morrissey $\mathrm{M}$, Johnson $\mathrm{BA}$, Wendt JK, et al. The rapid assessment of fatigue severity in cancer patients: use of the Brief Fatigue Inventory. Cancer. 1999 Mar; 85(5):1186-96.

21 Whitehead L. The measurement of fatigue in chronic illness: a systematic review of unidimensional and multidimensional fatigue measures. J Pain Symptom Manage. 2009 Jan; 37(1):107-28

22 Webster K, Cella D, Yost K. The Functional Assessment of Chronic Illness Therapy (FACIT) Measurement System: properties, applications, and interpretation. Health Qual Life Outcomes. 2003 Dec;1(1):79.

23 Chalder T, Berelowitz G, Pawlikowska T, Watts L, Wessely S, Wright D, et al. Development of a fatigue scale. J Psychosom Res. 1993;37(2): 147-53.

24 Lesage AC, Hagège $\mathrm{H}$, Tucat G, Gendre JP. Results of a national survey on quality of life in inflammatory bowel diseases. Clin Res Hepatol Gastroenterol. $2011 \mathrm{Feb}$;35(2):117-24.

25 Graff LA, Vincent N, Walker JR, Clara I, Carr R, Ediger J, et al. A population-based study of fatigue and sleep difficulties in inflammatory bowel disease. Inflamm Bowel Dis. 2011 Sep; 17(9):1882-9.

26 Banovic I, Gilibert D, Cosnes J. Crohn's disease and fatigue: constancy and co-variations of activity of the disease, depression, anxiety and subjective quality of life. Psychol Health Med. 2010 Aug;15(4):394-405.

27 Best WR, Becktel JM, Singleton JW, Kern F Jr. Development of a Crohn's disease activity index. National Cooperative Crohn's Disease Study. Gastroenterology. 1976 Mar;70(3): 439-44.

28 Walmsley RS, Ayres RC, Pounder RE, Allan RN. A simple clinical colitis activity index. Gut. 1998 Jul;43(1):29-32.

29 Gomollón F, Dignass A, Annese V, Tilg H, Van Assche G, Lindsay JO, et al.; ECCO. 3rd European Evidence-based Consensus on the Diagnosis and Management of Crohn's Disease 2016: Part 1: Diagnosis and Medical Management. J Crohn's Colitis. 2017 Jan; 11(1):3-25.

30 Sandborn WJ, Feagan BG, Hanauer SB, Lochs $\mathrm{H}$, Löfberg R, Modigliani R, et al. A review of activity indices and efficacy endpoints for clinical trials of medical therapy in adults with Crohn's disease. Gastroenterology. 2002 Feb; 122(2):512-30.
31 Higgins PD, Schwartz M, Mapili J, Zimmermann EM. Is endoscopy necessary for the measurement of disease activity in ulcerative colitis? Am J Gastroenterol. 2005 Feb;100(2): 355-61.

32 D'Haens G, Ferrante M, Vermeire S, Baert F, Noman M, Moortgat L, et al. Fecal calprotectin is a surrogate marker for endoscopic lesions in inflammatory bowel disease. Inflamm Bowel Dis. 2012 Dec;18(12):2218-24.

33 Panaccione R, Colombel JF, Louis E, PeyrinBiroulet L, Sandborn WJ. Evolving definitions of remission in Crohn's disease. Inflamm Bowel Dis. 2013 Jul;19(8):1645-53.

34 Langhorst J, Elsenbruch S, Koelzer J, Rueffer A, Michalsen A, Dobos GJ. Noninvasive markers in the assessment of intestinal inflammation in inflammatory bowel diseases: performance of fecal lactoferrin, calprotectin, and PMN-elastase, CRP, and clinical indices. Am J Gastroenterol. 2008 Jan;103(1): 162-9.

35 Vermeire S, Rutgeerts $P$. The changing face of treatment for Crohn's disease. Br J Surg. 2006 Apr;93(4):385-6.

36 von Roon AC, Karamountzos L, Purkayastha S, Reese GE, Darzi AW, Teare JP, et al. Diagnostic precision of fecal calprotectin for inflammatory bowel disease and colorectal malignancy. Am J Gastroenterol. 2007 Apr; 102(4):803-13.

37 Tibble JA, Sigthorsson G, Bridger S, Fagerhol MK, Bjarnason I. Surrogate markers of intestinal inflammation are predictive of relapse in patients with inflammatory bowel disease. Gastroenterology. 2000 Jul;119(1):15-22.

38 Freedman NS, Gazendam J, Levan L, Pack AI, Schwab RJ. Abnormal sleep/wake cycles and the effect of environmental noise on sleep disruption in the intensive care unit. Am J Respir Crit Care Med. 2001 Feb;163(2):451-7.

39 Muñoz M, Villar I, García-Erce JA. An update on iron physiology. World J Gastroenterol. 2009 Oct;15(37):4617-26.

40 R Core Team (2018). R: A language and environment for statistical computing. R Foundation for Statistical Computing, Vienna, Austria.Availableonlineathttps://www.R-project. org/.

41 Rietjens GJ, Kuipers H, Adam JJ, Saris WH, van Breda E, van Hamont D, et al. Physiological, biochemical and psychological markers of strenuous training-induced fatigue. Int $J$ Sports Med. 2005 Jan-Feb;26(1):16-26.

42 Craig A, Tran Y, Wijesuriya N, Boord P. A controlled investigation into the psychological determinants of fatigue. Biol Psychol. 2006 Apr;72(1):78-87.

43 Vogelaar L, de Haar C, Aerts BR, Peppelenbosch MP, Timman R, Hanssen BE, et al. Fatigue in patients with inflammatory bowel disease is associated with distinct differences in immune parameters. Clin Exp Gastroenterol. 2017 May; 10:83-90. 